\title{
INVENTARIO DE AUTOESTIMA PARA ADULTOS \\ ALPEL \\ FORMA - AD
}

\author{
Alejandro E. Loli Pineda \\ Ernestina López Vega
}

\section{RESUMEN}

Se presenta un Inventario de Autoestima para Adultos, concebido para el ámbito organizacional habiéndose considerado como componentes de la Autoestima a: Autoconocimiento, Autorrealización, Autorespeto y Autoconfianza. La forma definitiva es producto de una primera etapa de Criterio de Jueces y luego la validez y confiabilidad estadística. Es de aplicación colectiva e individual y presenta baremos para diferentes edades.

Palabras Clave: Autoestima, Inventario, Autorrealización, Autoconocimiento, Autorespeto, Autoconfianza.

\begin{abstract}
An Inventory of Self-esteem is presented for Adults, conceived for the organizational environment there being you considered as components of the Self-esteem to: selfrealization, self-knowledge, self-respect, and self-confidence. The definitive form is product of a first stage of Approach of Judges and the validity and statistical reliability. It is of collective application and singular and it presents scales for different ages.
\end{abstract}

Key Words: Self-esteem, Inventory, self-realization, self-knowledge, self-respect, selfconfidence. 


\section{ANTECEDENTES}

La autoestima como vivencia y experiencia existe desde que el hombre existe. En cambio como constructo científico surge a fines del siglo XIX, cuando se descubre que el "yo" se desdobla en "yo conocedor" y "yo conocido" (William J ames en Bonet, 1994); vale decir, cuando el ser humano descubre el "tu" como una experiencia distinta a él, durante el desarrollo de su estructura psíquica. Más tarde con el advenimiento de la psicoterapia humanista la autoestima adquiere un papel central en la autorrealización personal y en el tratamiento de los trastornos mentales (R.B. Burns,1990). De manera que aquello que parecía difícil o poco posible de ser medido se desvanece al encontrar que la persona es capaz de conocerse a sí mismo, tener un concepto, evaluarse aceptarse y respetarse, base sobre la cual se genera una percepción positiva o negativa de los demás. La autoestima en última instancia, es la "percepción evaluativa de uno mismo" (Bonet, 1994). De modo que, toda persona es capaz de expresar su manera de ser y actuar durante toda su vida en su interacción con el mundo y, por tanto, susceptible de ser medido como positiva o negativa, alta o baja, suficiente o deficiente. La autoestima es importante en la niñez y la adolescencia, pero también en la adultez y la vejez, así se ha entendido en la comunidad científica; sin embargo, se le dio mayor cobertura e importancia a la formación y el desarrollo de la autoestima en la niñez, preocupándose menos sobre las oportunidades que puede y debe tener el adulto de reconstruir, de reforzar o de mejorar su autoestima en el curso de su vida.

La referencia instrumental para la medición de la autoestima, más conocida en nuestro medio, la encontramos en el trabajo desarrollado por Coopersmith Stanley. en 1959, Y sólo publicado en 1967, lapso en el cual realiza investigaciones y tanteos que le permiten publicar una Escala para la medición de la autoestima de niños de $5^{\circ}$ y $6^{\circ}$ grado de estudios; la misma que fuera traducida al español por Panizo M.L (1988), y donde considera cuatro áreas o subescalas (yo general, yo social, académica escolar y hogar padres), a partir de cuatro criterios: el grado en que sienten que son amados y aceptados por aquellos que son importantes para ellos, capacidad para desempeñar tareas que consideran importantes, consecución de niveles morales y éticos y, finalmente, grado en que pueden influir en su vida y en la de los demás.

En la década de los 90, la globalización de la economía y la cultura trae consigo la búsqueda de la calidad en los servicios y los bienes que producen las organizaciones, a fin de lograr un desarrollo sostenido y competitivo; sin embargo, ni la tecnología ni el altruismo de los líderes empresariales son suficientes para el logro de dichos objetivos, se hace indispensable entonces considerar la presencia de la persona en este proceso de cambios. La cultura de la sociedad, del país, de las organizaciones y de las personas se pone en primer plano y con ella, los valores y la autoestima. Los valores son considerados como la clave del éxito organizacional (Peters,T. y Waterman,R.,1996;Rodriguez E,Mauro,1993) y la autoestima como la llave del éxito personal (Cruz Ramírez, J, 1997). Es cuando surge la necesidad de contar con un instrumento que permita medir la autoestima de la fuerza laboral y de la potencial mano de obra en las organizaciones. Se inicia entonces la construcción teórica sobre la forma cómo se constituyen los valores para la formación de "una cultura organizacional", llegando a la conclusión que éstos son producto de los valores adquiridos por las personas en la infancia y, la autoestima es la base de la formación de dichos valores personales y organizacionales (Loli Pineda, A. y López Vega, E., 1997), haciendo cada vez más necesaria la medición de la autoestima de los jóvenes y los adultos que forman parte o se incorporan a las organizaciones. Constructo teórico que conduce a la 
elaboración de reactivos que permitan conocer la autoestima de los integrantes de una organización y, llegado el momento, el aporte que una persona puede proporcionar al incorporarse a una organización. De tal modo que, los valores de las organizaciones se reconstruyan sobre bases sólidas de una autoestima positiva de sus miembros.

\section{PRIMERA PARTE}

\section{Construcción del Inventario de Autoestima}

Sigmund Freud (en Erik Erikson, 1950), Vandenbergh, (1963) y otros, consideran que la autoestima tiene sus orígenes entre los primeros días de nacido y los dos años, en la etapa de transición al pasar de la confianza habitual a la desconfianza, donde el niño recibe todo y no tiene capacidad para dar; de la comprensión y amor que los padres ofrecen al infante; de la estabilidad y continuidad de atenciones. Es decir, que al inclinarse hacia un desarrollo de plena confianza se va construyendo la fe, la esperanza, la aceptación de sí mismo y de los demás, así el niño se percibe importante y valioso, afianzando su autoestima. Mc Guigan (1974), afirma que la autoestima se basa en el mantenimiento de las relaciones objetales durante la niñez y contribuye a su vez a la formación del yo moral.

De esta manera, todas las investigaciones nos llevan a creer que la autoestima es un rasgo básico de la personalidad adquirida sobre la base de las interacciones sociales e influencias externas y consolidada con su propio comportamiento; de allí que una persona que se conoce es capaz de construir su escala de valores, ganar confianza y seguridad sobre sus actos, autorrealizarse y consecuentemente respetarse. Entonces, el objetivo es determinar el instrumento que permita medir éstas y otras variables que conforman la autoestima.

\section{CONSTRUCCION DEL INSTRUMENTO}

\section{Componentes de la Autoestima}

Los elementos que definen la autoestima tuvo su origen en las investigaciones desarrolladas por M. Rodriguez (s/f), quien percibe que la autoestima es producto del conocimiento, del concepto, de la evaluación, de la aceptación y del respeto que tiene una persona de sí misma; es decir, "si una persona se conoce y está consciente de sus cambios, crea su propia escala de valores y desarrolla sus capacidades, y si se acepta y respeta, tendrá autoestima. Por el contrario si una persona no se conoce, tiene un concepto pobre de sí misma, no se acepta ni respeta, entonces no tendrá autoestima". De este modo quedaba planteada la necesidad de orientar la elaboración de los ítems con base en el autoconocimiento, autoconcepto, autoevaluación, auto aceptación y autorrespeto; factores que deberían ser sometidas a prueba a fin de probar su validez en nuestra cultura, como componentes de la autoestima en nuestro medio.

\section{ELABORACION DE ÍTEMES}

Se elaboró ciento veinte ítemes a partir de la información recopilada de revistas, artículos, información teórica, boletines y todo tipo de documentos que ayudaran a definir 
los elementos que componen la autoestima y determinar los reactivos que permitan su medición.

\section{VALIDEZ DE CONTENIDO}

Entendido por validez de contenido como el grado con el cual se demuestra que una muestra es representativa del universo del cual proviene (Brown, 1996), o conjunto de reactivos relacionados de modo directo con el comportamiento o fenómeno que se quiere medir (G. Marín,1975), se elaboraron los ítemes en la cantidad antes señalada; los mismos que fueron sometidos a juicio de los expertos, quienes calificaron cada uno de los íemes de 1 a 5 puntos de acuerdo a la calidad; es decir, a mejor calidad mayor puntaje. La calidad se determinó sobre la base de tres criterios: que mida lo que se quiere medir, que la construcción del ítem cubra las normas mínimas de elaboración, y que la redacción sea lo más comprensible posible.

La ponderación de las calificaciones de los jueces nos llevó a una media mínima de 1.4 y máxima de 4.8 , permitiéndonos un promedio de 3.1 , constituyéndose éste puntaje en el mínimo aceptable para que un ítem tenga validez de contenido. De este modo, la versión original queda reducida a 46 ítemes. Correspondiendo 6 ítemes a autoconocimiento, 9 a autoconcepto, 12 a autoevaluación, 6 a autoaceptación y 13 ítemes a autorrespeto.

\section{ESTUDIO PILOTO}

Un análisis preliminar con los componentes de la autoestima, antes señalados, en una muestra de 3215 sujetos, arrojó un bajo nivel de confiabilidad, llevándonos a la necesidad de realizar un análisis estadístico de ítemes.

\begin{tabular}{|c|c|c|} 
Cuadro $\mathbf{N}^{\circ} \mathbf{1}$ \\
\begin{tabular}{|c|c|c|}
\hline Composición de la Muestra \\
\hline $\begin{array}{c}\text { Estudiantes } \\
\text { Universit. }\end{array}$ & $\begin{array}{c}\text { No Estudiantes } \\
\text { Egresad., Profeso }\end{array}$ & Total \\
\hline 2785 & 430 & 3215 \\
\hline
\end{tabular}
\end{tabular}

La muestra estuvo conformada por sujetos procedentes de Lima y provincias, de sexo masculino y femenino, de acuerdo a su estado civil y por grupos de edad.

\section{ANALISIS ESTADISTICO DE ÍTEMES}

El análisis factorial exploratorio nos permitió identificar otros factores, como componentes de la autoestima. Probablemente debido a las influencias de nuestro contexto socio cultural, los componentes señalados antes -de acuerdo con los resultados obtenidos por las investigaciones de M. Rodriguez- difieren de los resultados obtenidos en este análisis.

El análisis factorial exploratorio se efectuó utilizando el método de la Factorización Alpha por ser éste el método más efectivo para construir instrumentos, aplicándose posteriormente la Rotación Ortogonal (factores independientes) VARIMAX (kline,1993).

Los resultados presentados en el Cuadro $\mathrm{N}^{\circ} 2$ demuestran la existencia de cuatro factores importantes: el primero, que incluye a 16 ítemes y que permite explicar el $21.9 \%$ 
de la varianza total; el segundo, que incluye a 17 ítemes y que permite explicar el $16.9 \%$ de la varianza; el tercer factor, constituido por 5 ítemes y que explica el $12.4 \%$ de la varianza y, el cuarto, factor que incluye a 3 ítemes que explican el $8.4 \%$ de la varianza. Estos cuatro factores en conjunto explican el $59.6 \%$ de la varianza total.

También es necesario indicar que los ítemes 3, 17, 23, 29 Y 37, no han sido incluidos en ningún factor por alcanzar pesos factoriales inferiores a 0.20 .

\section{Cuadro $\mathrm{N}^{\circ} 2$}

Análisis Factorial exploratorio de los itemes del “AlPEL” forma-AD

\begin{tabular}{|c|c|c|c|c|c|c|c|c|c|}
\hline Itemes & Fact.1 & Fact.2 & Fact.3 & Fact.4 & Ítemes & Fact.1 & Fact.2 & Fact.3 & Fact.4 \\
\hline 1 & & & .35 & & 25 & & .40 & & \\
2 & & .40 & & & 26 & .53 & & & \\
4 & .26 & & & & 27 & .75 & & & \\
5 & .51 & & & & 28 & & .54 & & \\
6 & & .41 & & & 30 & & & .39 & \\
7 & & .38 & & & 31 & .63 & & & \\
8 & .66 & & & & 32 & & & & .41 \\
9 & & & .38 & & 33 & & .40 & & \\
10 & & .41 & & & 34 & & .39 & & .49 \\
11 & & .46 & & & 35 & & & & \\
12 & & .30 & & & 36 & & & .41 & \\
13 & & .40 & & & 38 & & .36 & & \\
14 & & .55 & & & 39 & & & .48 & \\
15 & .45 & & & & 40 & .70 & & & \\
16 & & .39 & & & 41 & .57 & & & \\
19 & .63 & & & & 42 & & & & \\
20 & .39 & & & & 43 & .51 & & & .53 \\
21 & & 39 & & & 44 & & .43 & & \\
22 & .48 & & & & 45 & .77 & .29 & & \\
24 & .70 & & & & 46 & & & & \\
\hline
\end{tabular}

Varianza Explicada: $21.9 \% \quad 16.9 \% \quad 12.4 \% \quad 8.4 \%$

$N=3215$

Nota:Los ítemes 3,17,23,29,y 37 no se incluyen por no presentar pesos factoriales relevantes

\section{DENOMINACION ACTUAL DE LOS FACTORES}

Factor 1: Autoconocimiento. El yo es una parte de la estructura psíquica de toda persona, que tiene necesidades, habilidades y aspiraciones, que siente y actúa en armonía con los estímulos del medio ambiente del cual es consciente. Conocer sus derechos y obligaciones, sus sentimientos de satisfacción y de confianza le permiten orientar su vida, controlar sus impulsos, crear mecanismos de defensa y seguridad personal, haciendo que el sujeto logre una personalidad integrada y fuerte.

Factor 2: Autorrealización. Implica el cumplimiento de metas y aspiraciones en el 
aprendizaje y actualización, en la ejecución de tareas en el trabajo, en la libertad para expresar sentimientos, en la aceptación y cumplimiento de roles sexuales, en los éxitos logrados por su actuación, en su capacidad para la autocrítica, en la simpatía que reflejan sus actos, en la defensa de sus derechos y obligaciones, en el aprecio y respeto que otorga a los demás; de manera que la persona que hace conciencia de sus propios actos incrementa su autorrealización, elevando su autoestima.

Factor 3: Autorrespeto. Refleja esencialmente la expresión y manejo adecuado de sus sentimientos y emociones sin causar daño a los demás, evitándose sentimientos de culpa; situación que le hace sentir único y diferente de los demás.

Factor 4: Autoconfianza. Es la expresión de sí mismo reflejada en la conducta frente a los demás, reconociendo en ellos la expresión de sus sentimientos con libertad.

\begin{tabular}{|l|l|}
\hline \multicolumn{1}{|c|}{ Factores } & \multicolumn{1}{|c|}{ Itemes } \\
\hline Autoconocimiento & $4,5,8,15,18,19,20,22,24,26,27,31,40,41,43,45$ \\
\hline Autorrealización & $2,6,7,10,11,12,13,14,16,21,25,28,33,34,18,44,46$ \\
\hline Autorrespeto & $1,9,30,36,39$ \\
\hline Autoconfianza & $32,35,42$ \\
\hline
\end{tabular}

\section{ANALSIS PSICOMETRICO y CONFIABILIDAD DE CADA ESCALA}

El puntaje de una escala es confiable cuando tenemos razones para creer que dicho puntaje es estable y fide-digno. Es decir, hasta qué punto el puntaje es un índice "apto", que está libre de error atribuible a la casualidad (H. E. Garrett, 1974); Y que las aplicaciones repetidas de una misma medida a un mismo grupo de $\neg$ ben arrojar resultados similares, de manera que las variaciones en los resultados deben ser congruentes al cambiar el grupo (G.Marín,1975).

Factor 1: En el Cuadro $\mathrm{N}^{\circ} 3$ observamos que el análisis de la correlación de Ítem Test corregida indica que todos los ítemes cumplen con el criterio. Es decir, la correlación es mayor de 0.20; observándose que los valores fluctúan entre 0.34 (ítem 20) hasta el 0.68 (item 45).

Factor 2: Autorrealización. Implica el cumplimiento de metas y aspiraciones en el aprendizaje y actualización, en la ejecución de tareas en el trabajo, en la libertad para expresar sentimientos, en la aceptación y cumplimiento de roles sexuales, en los éxitos logrados por su actuación, en su capacidad para la autocrítica, en la simpatía que reflejan sus actos, en la defensa de sus derechos y obligaciones, en el aprecio y respeto que otorga a los demás; de manera que la persona que hace conciencia de sus propios actos incrementa su autorrealización, elevando su autoestima.

Factor 3: Autorrespeto. Refleja esencialmente la expresión y manejo adecuado de sus sentimientos y emociones sin causar daño a los demás, evitándose sentimientos de culpa; situación que le hace sentir único y diferente de los demás. 


\section{Cuadro $\mathbf{N}^{\circ} \mathbf{3}$}

Análisis psicométrico y confiabilidad de la escala de autoconocimiento de «ALPEL» Forma - $A D$

\begin{tabular}{|c|c|c|c|}
\hline Ítem & Media & DS. & ritc \\
\hline 4 & .63 & .48 & $.41^{*}$ \\
5 & .84 & .37 & $.48^{*}$ \\
8 & .88 & .33 & $.62^{*}$ \\
45 & .88 & .32 & $.68^{*}$ \\
27 & .90 & .30 & $.67^{*}$ \\
24 & .87 & .33 & $.67^{*}$ \\
40 & .84 & .37 & $.63^{*}$ \\
31 & .83 & .38 & $.56^{*}$ \\
19 & .82 & .38 & $.61^{*}$ \\
26 & .77 & .42 & $.54^{*}$ \\
43 & .78 & .42 & $.47^{*}$ \\
22 & .75 & .43 & $.52^{*}$ \\
15 & .77 & .42 & $.47^{*}$ \\
18 & .70 & .46 & $.44^{*}$ \\
20 & .76 & .43 & $.34^{*}$ \\
\hline \multicolumn{4}{|c|}{ Kuder Richardson $20=0.88$} \\
\hline
\end{tabular}

(*) Cumple el criterio $N=3213$

Cuadro $\mathrm{N}^{\circ} 4$

Análisis psicométrico y confiabilidad de la escala de autorrealización de «ALPEL» Forma - AD

\begin{tabular}{|r|c|c|c|}
\hline Ítem & Media & D.S. & ritc \\
\hline 14 & .89 & .32 & $.47^{*}$ \\
28 & .85 & .35 & $.47^{*}$ \\
11 & .86 & .35 & $.41^{*}$ \\
44 & .94 & .24 & $.34^{*}$ \\
10 & .77 & .42 & $.35^{*}$ \\
6 & .86 & .34 & $.35^{*}$ \\
13 & .94 & .23 & $34^{*}$ \\
25 & .91 & .28 & $.29^{*}$ \\
33 & .89 & .32 & $.28^{*}$ \\
2 & .91 & .29 & $.24^{*}$ \\
21 & .94 & .24 & $.25^{*}$ \\
16 & .87 & .34 & $.22^{*}$ \\
34 & .96 & .19 & $23^{*}$ \\
7 & .91 & .29 & $.23^{*}$ \\
38 & .95 & .22 & $23^{*}$ \\
12 & .99 & .12 & $.21^{*}$ \\
6 & .97 & .18 & $.22^{*}$ \\
\hline \multicolumn{3}{|c|}{ Kuder-Richardson $20=0.71$} \\
\hline
\end{tabular}

(*) Cumple el criterio $N=3212$

\section{Cuadro $\mathrm{N}^{\circ} 5$}




\section{Análisis psicométrico y confiabilidad de la escala de autorespeto de «ALPEL»Forma-AD}

\begin{tabular}{|c|c|c|c|}
\hline Item & Media & D.s. & ritc \\
\hline 39 & .52 & .50 & $.29^{*}$ \\
36 & .62 & .49 & $.30^{*}$ \\
30 & .90 & .29 & $.20^{*}$ \\
9 & .40 & .49 & .10 \\
1 & .66 & .48 & .13 \\
\hline \multicolumn{4}{|c|}{ Kuder-Richardson $20=0.40$} \\
\hline
\end{tabular}

(*) Cumple el criterio $N=3214$

Factor 4: En el Cuadro $\mathrm{N}^{\circ} 6$ observamos que el análisis de la correlación de Ítem Test Corregida indica que casi todos los ítemes cumplen con el criterio. Es decir, la correlación es mayor de 0.20 en conjunto; observándose que los valores fluctúan entre 0.12 (item 32) hasta el 0.42 (ítem 35).

La confiabilidad obtenida a través del método de la consistencia interna con base en el coeficiente Kuder Richardson 20, en conjunto, es de 0.47 , lo que nos permite indicar que los puntajes obtenidos con esta Escala son confiables.

\section{Cuadro $\mathrm{N}^{\circ} 6$}

Análisis psicométrico y confiabilidad de la escala de autorrealización de «ALPEL» Forma $-A D$

\begin{tabular}{|c|c|c|c|}
\hline Item & Media & D.S. & ritc \\
\hline 42 & .58 & .49 &. $.35^{*}$ \\
35 & .63 & .48 & $.42^{*}$ \\
32 & .57 & .50 & .12 \\
\hline \multicolumn{4}{|c|}{ Kuder-Richardson $20=0.47$} \\
\hline
\end{tabular}

$(*)=$ Cumple el criterio $N=3214$

En conclusión, el análisis de la consistencia de todos los ítemes que conforman el "ALPEL" - Forma AD, obtiene un coeficiente de confiabilidad Kuder Richardson 20 de 0.84 , lo que nos indica que la prueba permite obtener puntajes confiables.

\section{VALIDEZ DE CONSTRUCTO}

La validez de constructo se logró a través del análisis factorial, donde las intercorrelaciones de un número de test se examinan y, de ser posible, se explican en términos de un número mucho menor de "factores o categorías características más generales. Los factores abarcan presuntamente todos los aspectos medidos por los test individuales. A veces se encuentran, por ejemplo, que 3 ó 4 factores explican las intercorrelaciones obtenidas entre 15 o más test. La validez de un test determinado se define por sus pesos factoriales, y éstos son dados por la correlación del test con cada factor (H. E. Garrett, 1974).

Inicialmente, en el presente caso, se realizó un Análisis Factorial Exploratorio de ítemes que originó la clasificación en cuatro Factores, los cuales en sus análisis respectivos demostraron no sólo tener un peso aceptable, sino que la agrupación mostró tener sentido y consistencia, vale decir, confiabilidad. A continuación se trató de estudiar la existencia de 
factores de segundo orden, el mismo fue efectuado con los puntajes obtenidos por los sujetos. Los resultados obtenidos (ver Cuadro $\mathrm{N}^{\circ} 7$ ), indican la presencia de un sólo factor que permite explicar el $37.60 \%$ de la varianza total. Esto nos indica que estos elementos estarían conformando de forma adecuada el constructo de la autoestima en nuestro medio.

\section{Cuadro $\mathrm{N}^{\circ} 7$}

Análisis factorial de las sub-escalas del «ALPEL»Forma - AD

\begin{tabular}{|l|c|c|c|}
\hline Factor & Mediana & D.S. & Factor 1 \\
\hline Factor 1 & 12.80 & 3.78 & 0.71 \\
Factor 2 & 15.39 & 2.07 & 0.68 \\
Factor 3 & 3.09 & 1.23 & 0.56 \\
Factor 4 & 1.78 & 1.02 & 0.49 \\
\hline \multicolumn{4}{|c|}{ Varianza explicativa $=37.60 \%$} \\
\hline \multicolumn{3}{|c|}{ Medida de adecuación del muestreo de } \\
Kaiser-Meyer-Olkin =0.61 \\
Test de esfericidad de Bartlett=516,353 \\
\hline
\end{tabular}

\section{FUNCIONAMIENTO DEL INSTRUMENTO}

El Inventario de Autoestima para Adultos, ALPEL, Forma-AD, es una prueba de personalidad que explora la manera de ser y de actuar de una persona en su vida cotidiana, base sobre la cual, supone también su actuación en la organización. Está compuesta por 41 ítemes de respuestas dicotómicas SI o No es su forma de ser o actuar. Estas respuestas califican la Autoestima Alta o Baja respectivamente, dentro de un continuo, según su ubicación en la clave de calificación.

\section{INSTRUCCIONES PARA LA APLICACIÓN}

\section{CARACTERISTICAS BASICAS}

\section{Datos Generales:}

ALPEL Forma-AD es un inventario flexible a las necesidades $\mathrm{u}$ objetivos del administrador de la prueba y de la forma de aplicación.

\section{Forma de aplicación}

ALPEL Forma - AD es un inventario que se puede administrar en forma individual o colectiva. Nuestra experiencia ha sido en ambas formas, demostrando su operatividad sin dificultad alguna.

\section{Tiempo de duración de la prueba}

ALPEL Forma - AD es una prueba sencilla y rápida. Su duración promedio es 15 minutos.

\section{Características de presentación}

El inventario consta de un cuadernillo de preguntas que va acompañado de hojas de respuestas (protocolo), los mismos que son entregados a los examinados. 


\section{Instrucciones}

Las instrucciones de ALPEL Forma-AD se encuentran en la primera carilla del cuadernillo. Estas serán leídas por el examinador, sea su aplicación individual o colectiva, a fin de hacer alguna aclaración si es preciso. El enunciado es el siguiente:

"En este cuadernillo no existen respuestas correctas o incorrectas, ni respuestas malas o buenas. Se trata de mirarse interiormente o percibirse a sí mismos para contestar todas las preguntas que se presentan. Responda tan rápido como sea posible, sin pensar demasiado. Todas sus respuestas son válidas. Normalmente la primera idea que se nos viene a la mente es la más apropiada. Sea honesto consigo mismo. Se guardará especial reserva a sus respuestas.

A continuación encontrará una serie de frases u oraciones. Lea cada una de ellas y conteste colocando un aspa $(X)$ en la respuesta -SI o NO-que haya escogido".

Por ejemplo:

- Soy una persona simpática.

- Maltrato a los demás como lo hacen conmigo.

- Siempre hago buenas acciones

$\begin{array}{cc}\text { SI } & \text { NO } \\ & X \\ & X\end{array}$

$X$

\section{NORMAS PARA LA CALIFICACION Y PUNTUACION}

La calificación se puede hacer siguiendo la clave de respuestas o mediante el uso de plantillas. Cada estímulo resuelto adecuadamente es calificado con 1 punto y estímulo mal resuelto se califica con $\mathrm{O}$.

\section{CALIFICACION DIRECTA MEDIANTE CLAVE:}

Se trabaja ubicando la respuesta del examinado en la Clave de Calificación y puntuando con 1 si es Alta y $\mathrm{O}$ si es Baja. El puntaje total es de 41 puntos.

\section{CLAVE DE CALIFICACION}

\begin{tabular}{|c|c|c|c|c|c|c|c|c|c|c|c|}
\hline \multicolumn{2}{|c|}{ AUTOESTIMA } & \multicolumn{2}{c|}{ AUTOESTIMA } & \multicolumn{3}{c|}{ AUIDESTIMA } & \multicolumn{3}{|c|}{ AUTOESTIMA } \\
\hline $\mathbf{N}^{\circ}$ & ALTA & BAJA & $\mathbf{N}^{\circ}$ & ALTA & BAJA & $\mathbf{N}^{\circ}$ & ALT & BAJA & $\mathbf{N}^{\circ}$ & ALT & BAJA \\
\hline \multirow{2}{*}{1} & SI & NO & 11 & SI & NO & 21 & NO & SI & 31 & SI & NO \\
2 & SI & NO & 12 & SI & NO & 22 & SI & NO & 32 & SI & NO \\
3 & NO & SI & 13 & SI & NO & 23 & NO & SI & 33 & SI & NO \\
4 & NO & SI & 14 & NO & SI & 24 & NO & SI & 34 & NO & SI \\
5 & SI & NO & 15 & SI & NO & 25 & SI & NO & 35 & NO & SI \\
6 & SI & NO & 16 & NO & SI & 26 & SI & NO & 36 & NO & SI \\
7 & NO & SI & 17 & NO & SI & 27 & NO & SI & 37 & NO & SI \\
8 & SI & NO & 18 & NO & SI & 28 & SI & NO & 38 & NO & SI \\
9 & SI & NO & 19 & SI & NO & 29 & SI & NO & 39 & SI & NO \\
10 & SI & NO & 20 & NO & SI & 30 & SI & NO & 40 & NO & SI \\
& & & & & & & & & SI & NO \\
\hline
\end{tabular}

Las palabras "SI" o "NO" califican lo que corresponde (Alta o Baja), según su ubicación en la Clave de Calificación. Por ejemplo: si el ítem es negativa a la persona y es respondida como "SI", se califica como autoestima baja (O puntos), pero si es positiva a la persona y es respondida como "SI", se califica como autoestima alta (1 punto). Si por el 
contrario, el ítem es negativa a la persona y es respondida como "NO", se califica como autoestima alta (1 punto), pero si es positiva a la persona y es respondida como "NO", se califica como autoestima baja (0 puntos)

\begin{tabular}{|c|c|c|}
\hline Ítemes & Rpta. & Grado de Autoestima \\
\hline Negativa & SI & Autoestima Baja \\
Positiva & SI & Autoestima Alta \\
Negativa & NO & Autoestima Alta \\
Positiva & NO & Autoestima Baja \\
\hline
\end{tabular}

Entre la respuesta "SI" y la respuesta "NO" existe una escala o continuo que va de 1 a 5 , y que representa el puntaje bruto, que convertido en percentil es calificada como categoría o diagnóstico.

$\begin{array}{ccccc}\text { Muy Baja } & \text { Baja } & \text { Intermedia } & \text { Alta } & \text { Muy Alta } \\ 2 & 2 & 3 & 4 & 5\end{array}$

\section{CALIFICACION MEDIANTE PLANTILLA}

Cuando se usa la plantilla se coloca ésta sobre el protocolo u hoja de respuestas del examinado, tratando de hacer coincidir los puntos de referencia.

Se califica con 1 punto todas aquellas respuestas que resaltan sobre la plantilla (estos son los aciertos). Cada sub-escala permite un puntaje parcial, para obtener luego el puntaje total de 41 puntos.

Nota Importante.- Al convertir los puntajes en percentiles, por el momento es preferible usar los puntajes totales y no así los que corresponden a los puntajes de los factores por cuanto éstos están en fase de experimentación.

\section{PUNTUACION POR FACTORES}

Sub-Escala

Autoconocimiento

Autorrealización

Autorrespeto

Autoconfianza

Total
Itemes

$4,5,8,15,18,19,20,22,24,26,27,31,40,41,43,45$

$2,6,7,10,11,12,13,14,16,21,25,28,33,34,18,44,46$

$1,9,30,36,39$

$32,35,42$
Ptje. Máximo

16

17

5

3

41

\section{NORMAS INTERPRETATIVAS}

\section{Conversión de puntajes directos en percentiles}

El puntaje bruto total obtenido mediante la suma de los aciertos (mediante el uso directo de las Claves de Calificación o mediante el uso de la plantilla) es llevado al Baremo o Escala para ser convertido en Percentiles y la categoría di agnóstica respectiva.

Este proceso consiste en ubicar la columna "Autoestima total" en los Baremos donde están agrupados los puntajes de 1 a 41, se busca el puntaje total que corresponde al 
examinado, se ubica el percentil que le corresponde en la columna de los "Percentiles" y luego el "Nivel" y el "Diagnóstico". (Teniendo en cuenta que los factores que componen la autoestima están en proceso de experimentación no es conveniente, por ahora, hacer uso de los puntajes parciales para obtener los percentiles)

\section{INTERPRETACION DE LA AUTOESTIMAGLOBAL}

"Solo se podrá respetar a los demás cuando se respete uno a sí mismo; sólo podremos dar cuando nos hemos dado a nosotros mismos, sólo podemos amar cuando nos amemos a nosotros mismos" (Maslow, 1979)

Define a la persona con autoestima.

\section{ALTA}

Persona de convicciones y principios, de valores positivos, capaz de conocer sus derechos y obligaciones, sus sentimientos de satisfacción y descontento, de confianza y desconfianza; de manera que pueda orientar su vida hacia la realización de sus aspiraciones, respetando lo que corresponde a los demás, reconociendo y corrigiendo sus errores, por los cuales se siente único y confiado.

\section{BAJA}

Persona insegura, de escasos principios y débiles, de valores negativos. No reconoce los derechos ni los sentimientos de los demás; desconfiado, inseguro, incapaz de orientar su vida hacia metas positivas sin causar daño a los demás; utiliza cualquier medio para lograr sus aspiraciones.

\section{INTERPRETACION DE LOS FACTORES DE LA AUTOESTIMA}

FACTOR 1: Autoconocimiento "Cuando aprendemos a conocemos en verdad vivimos". (R. Schüller)

Define a la persona que se conoce así mismo.

\section{ALTA}

Es consciente de sus actos, se adapta a los cambios, conoce sus derechos y obligaciones, es capaz de relacionarse con madurez con los demás -incluso con el sexo opuesto. Controla bien sus impulsos, asequible ante la autoridad. Seguro confiado, capaz de orientar su vida hacia metas positiva y claras. de personalidad y fuerte.

\section{BAJA}

Persona con escasa capacidad para reconocer sus actos, sus derechos, obligaciones y necesidades; duro e inflexible a los cambios. Sus relaciones interpersonales son difíciles y generalmente inmaduras, especialmente frente al sexo opuesto. Impulsivo, agresivo. Inseguro y desconfiado a los demás, es incapaz de orientar su vida hacia metas claramente diseñadas.

\section{FACTOR 2: Autorrealización}

" ... Sólo podremos dar cuando nos hemos dado a nosotros mismo ... ". (A. Maslow, 1979) 
Define a la persona que se realiza en sus metas y aspiraciones

\begin{abstract}
ALTA
Persona principista, amante de los valores como la verdad, la belleza, la justicia; De una riqueza interior insospechada, capaz de alcanzar realización y crecimiento psicológico en cada uno de sus actos (en la actualización de conocimientos, en la satisfacción con el trabajo que realiza, en la libertad e individualidad de sentimientos, aceptación de su sexualidad, etc.). Consciente de los cambios, es capaz de autoevaluación, de rectificación de errores y aprendizaje de ellos. Tiene confianza, capaz de tomar decisiones trascendentes; aprecia los demás y recibe simpatía; lucha por sus derechos y es responsable de sus obligaciones.
\end{abstract}

\title{
BAJA
}

Persona de escasos principios, generalmente apegado a valores negativos, busca sacar ventaja a las oportunidades para cubrir necesidades inmediatas, le importa poco el futuro, siempre insatisfecho y frustrado. No es consciente de los cambios, es incapaz de autocrítica y rectificación. Es desconfiado, incapaz de tomar decisiones importantes; no se aprecia ni se respeta, tampoco a los demás. Es irresponsable y no distingue el derecho de los demás.

\section{FACTOR 3: Autorrespeto}

"Solo se podrá respetar a los demás cuando uno se respete uno así mismo ... ". (Maslow)

"La autoestima es un silencioso respeto por uno mismo". (Elkkins)

Define a la persona que se respeta a sí mismo.

\section{ALTA}

Persona que sabe atender sus propias necesidades y valores, que es capaz de manejar adecuadamente sus pensamientos, sentimientos y sus actos. Sin causarse daño ni expresar hostilidad a los demás. Evita emitir juicio sobre las personas, aunque sí opina sobre los actos de ellos. Sintiéndose por eso, único y diferente de los demás.

\section{BAJA}

Persona que descuida sus propias necesidades y valores, que maneja en forma inconveniente sus sentimientos, pensamientos y actos, sin importarle el daño que se puede causar a sí mismo y a los demás. Siempre emitiendo juicio sobre las personas más que sobre los actos de ellos.

\section{FACTOR 4: Autoconfianza}

«... al inclinarse hacia un desarrollo de plena confianza se va construyendo la fe, la esperanza, la aceptación de sí mismo y de los demás; así la persona se percibe importante y valioso, afianzando su autoestima».

Define a la persona que tiene confianza de sí mismo. 


\section{ALTA}

Persona segura, que tiene fe en sus actos, que se percibe importante y valioso, que le permite despegar y crecer por la confianza que se tiene. Capaz de aceptar la expresión de los sentimientos de los demás con libertad porque confía en ellos.

\section{BAJA}

Persona insegura, que no tiene fe en sus actos ni en los de otros; se percibe desvalido y limitado frente a los retos o carece de interés por desarrollarse y crecer. Es incapaz de aceptar la expresión de los sentimientos ajenos con libertad porque desconfía de ellos.

\section{BAREMOS}

Tabla $\mathbf{N}^{0} \mathbf{1}$

Baremo de ALPEL para estudiantes

\begin{tabular}{|c|c|c|c|c|c|c|c|}
\hline Pc & $\begin{array}{c}\text { Autoestima } \\
\text { Total }\end{array}$ & $\begin{array}{c}\text { Factor I } \\
\text { Autocon. }\end{array}$ & $\begin{array}{c}\text { Factor } \\
\text { 2Autorreal. }\end{array}$ & $\begin{array}{c}\text { Factor 3 } \\
\text { Autorres. }\end{array}$ & $\begin{array}{c}\text { Factor 4 } \\
\text { autonconf. }\end{array}$ & $\begin{array}{c}\text { Nivel } \\
\text { Diagn }\end{array}$ & Categoría \\
\hline $0-20$ & $0-30$ & 0.12 & $0-14$ & $0-1$ & 0 & I & Aut.muy alta \\
$21-40$ & $31-33$ & 13 & 15 & 2 & 1 & II & Aut.Baja \\
$41-60$ & $34-36$ & $14-15$ & 16 & 3 & 2 & III & Aut.Intermedia \\
$61-80$ & 37 & & & 4 & & IV & Aut.Alta \\
$81-99$ & $38-40$ & 16 & 17 & 5 & 3 & V & Aut.muy alta \\
& & & & & & & \\
\hline
\end{tabular}

$X=33.34 \quad$ D.S. $=7.30 \quad N=430$

Tabla $\mathbf{N}^{\circ} 2$

Baremo de ALPEL para no estudiar

\begin{tabular}{|c|c|c|c|c|c|c|c|}
\hline Pc & $\begin{array}{c}\text { Autoestima } \\
\text { Total }\end{array}$ & $\begin{array}{c}\text { Factor } \\
\text { Autocon. }\end{array}$ & $\begin{array}{c}\text { factor II } \\
\text { Autorreal. }\end{array}$ & $\begin{array}{c}\text { Factor } \\
\text { III } \\
\text { Autorres. }\end{array}$ & $\begin{array}{c}\text { Factor 4 } \\
\text { autonconf. }\end{array}$ & $\begin{array}{c}\text { Nivel } \\
\text { Diagn }\end{array}$ & Categoría \\
\hline $0-20$ & $0-23$ & $0-1$ & $0-15$ & $0-2$ & 0 & I & Aut.muy alta \\
$21-40$ & $24-28$ & $2-8$ & 16 & 3 & 1 & II & Aut.Baja \\
$41-60$ & $29-36$ & $9-14$ & & 4 & 2 & III & Aut.Intermedia \\
$61-80$ & $37-39$ & 15 & & & 3 & VV & Aut.Alta \\
$81-99$ & $40-41$ & 16 & 17 & 5 & Aut.muy alta \\
\hline
\end{tabular}

$X=31.30 \quad$ D.S. $=7.30 \quad N=430$

Tabla $\mathbf{N}^{\mathbf{3}} 3$

Baremo de ALPEL por procedencia_Lima

\begin{tabular}{|c|c|c|c|c|c|c|c|}
\hline Pc & $\begin{array}{c}\text { Autoestima } \\
\text { Total }\end{array}$ & $\begin{array}{c}\text { Factor } \\
\text { Autocon. }\end{array}$ & $\begin{array}{c}\text { factor II } \\
\text { Autorreal. }\end{array}$ & $\begin{array}{c}\text { Factor III } \\
\text { Autorres. }\end{array}$ & $\begin{array}{c}\text { Factor 4 } \\
\text { autonconf. }\end{array}$ & $\begin{array}{c}\text { Nivel } \\
\text { Diagn }\end{array}$ & Categoría \\
\hline $0-20$ & $0-29$ & $0-12$ & $0-13$ & $0-2$ & 0 & I & Aut.muy alta \\
$21-40$ & $30-33$ & $13-14$ & 14 & 3 & 1 & II & Aut.Baja \\
$41-60$ & $34-36$ & 15 & 16 & & 2 & III & Aut.Intermedia \\
$61-80$ & $37-38$ & & & 4 & & IV & Aut.Alta \\
$81-99$ & $39-41$ & 16 & 17 & 5 & 3 & V & Aut.muy alta \\
\hline
\end{tabular}

$X=33.30$ D.S. $=5.30 \quad N=2548$ 
Tabla $\mathbf{N}^{\circ} 4$

Baremo de ALPEL por procedencia-Provincia

\begin{tabular}{|c|c|c|c|c|c|c|c|}
\hline Pc & $\begin{array}{c}\text { Autoestima } \\
\text { Total }\end{array}$ & $\begin{array}{c}\text { Factor } \\
\text { Autocon. }\end{array}$ & $\begin{array}{c}\text { factor II } \\
\text { Autorreal. }\end{array}$ & $\begin{array}{c}\text { Factor III } \\
\text { Autorres. }\end{array}$ & $\begin{array}{c}\text { Factor 4 } \\
\text { autonconf. }\end{array}$ & $\begin{array}{c}\text { Nivel } \\
\text { Diagn }\end{array}$ & Categoría \\
\hline $0-20$ & $0-26$ & $0-8$ & $0-15$ & $0-2$ & 0 & I & Aut.muy alta \\
$21-40$ & $37-32$ & $9-13$ & 16 & 3 & 1 & II & Aut.Baja \\
$41-60$ & $33-35$ & 14 & & & 2 & III & Aut.Intermedia \\
$61-80$ & $36-37$ & 15 & 17 & 4 & & IV & Aut.Alta \\
$81-99$ & $38-40$ & 16 & & 5 & 3 & V & Aut.muy alta \\
\hline
\end{tabular}

$X=32.18 \quad$ D.S. $=5.91 \quad N=667$

Tabla $\mathbf{N}^{0} 5$

Baremo de ALPEL por Sexo-Varones

\begin{tabular}{|c|c|c|c|c|c|c|c|}
\hline Pc & $\begin{array}{c}\text { Autoestima } \\
\text { Total }\end{array}$ & $\begin{array}{c}\text { Factor } \\
\text { Autocon. }\end{array}$ & $\begin{array}{c}\text { factor II } \\
\text { Autorreal. }\end{array}$ & $\begin{array}{c}\text { Factor III } \\
\text { Autorres. }\end{array}$ & $\begin{array}{c}\text { Factor3 } \\
\text { Autoconf. }\end{array}$ & $\begin{array}{c}\text { Nivel } \\
\text { Diagn }\end{array}$ & Categoría \\
\hline $0-20$ & $0-28$ & $0-11$ & $0-13$ & $0-2$ & 0 & I & Aut.muy alta \\
$21-40$ & $29-33$ & $12-13$ & 14 & & 1 & II & Aut.Baja \\
$41-60$ & $34-35$ & $14-15$ & $15-16$ & & 2 & III & Aut.Intermedia \\
$61-80$ & $36-38$ & & & & IV & Aut.Alta \\
$81-99$ & $39-41$ & 16 & 17 & & 3 & V & Aut.muy alta \\
\hline
\end{tabular}

Tabla $\mathbf{N}^{\circ} 6$

Baremo de ALPEL para estudiantes

\begin{tabular}{|l|l|l|l|l|l|l|l|}
\hline Pc & $\begin{array}{l}\text { Autoestima } \\
\text { Total }\end{array}$ & $\begin{array}{l}\text { Factor I } \\
\text { Autocon. }\end{array}$ & $\begin{array}{l}\text { Factor } \\
\text { 2Autorreal. }\end{array}$ & $\begin{array}{l}\text { Factor 3 } \\
\text { Autorres. }\end{array}$ & $\begin{array}{l}\text { Factor 4 } \\
\text { autonconf. }\end{array}$ & $\begin{array}{l}\text { Nivel } \\
\text { Diagn }\end{array}$ & Categoría \\
\hline $0-20$ & $0-30$ & $0-12$ & $0-14$ & $0-1$ & 0 & I & Aut.muy alta \\
$21-40$ & $31-33$ & 13 & $15-16$ & 2 & 1 & II & Aut.Baja \\
$41-60$ & $34-36$ & $14-15$ & & 3 & 2 & III & Aut.Intermedia \\
$61-80$ & 37 & & & 4 & & IV & Aut.Alta \\
$81-99$ & $38-40$ & 16 & 17 & 5 & 3 & V & Aut.muy alta \\
\hline
\end{tabular}

$X=33.37 \quad$ D.S. $=5.28$

Tabla $\mathbf{N}^{\circ} 7$

Baremo de ALPEL por edad-18-20años

\begin{tabular}{|c|c|c|c|c|c|c|c|}
\hline Pc & $\begin{array}{c}\text { Autoestima } \\
\text { Total }\end{array}$ & $\begin{array}{c}\text { Factor } \\
\text { Autocon. }\end{array}$ & $\begin{array}{c}\text { factor II } \\
\text { Autorreal. }\end{array}$ & $\begin{array}{c}\text { Factor } \\
\text { III } \\
\text { Autorres. }\end{array}$ & $\begin{array}{c}\text { Factor3 } \\
\text { Autoconf. }\end{array}$ & $\begin{array}{c}\text { Nivel } \\
\text { Diagn }\end{array}$ & Categoría \\
\hline $0-20$ & $0-30$ & $0-12$ & $0-14$ & $0-1$ & 0 & I & Aut.muy alta \\
$21-40$ & $31-33$ & 13 & 15 & 2 & 1 & II & Aut.Baja \\
$41-60$ & $34-35$ & $14-15$ & 16 & 3 & 2 & III & Aut.Intermedia \\
$61-80$ & $36-37$ & & & 4 & & IV & Aut.Alta \\
$81-99$ & $38-40$ & 16 & 17 & 5 & 3 & V & Aut.muy alta \\
\hline
\end{tabular}

$X=33.34 \quad$ D.S. $=4.96 \quad N=1259$ 


\section{Tabla $\mathbf{N}^{\circ} 8$}

Baremo de ALPEL por edad 21-30 años

\begin{tabular}{|c|c|c|c|c|c|c|c|}
\hline Pc & $\begin{array}{c}\text { Autoestima } \\
\text { Total }\end{array}$ & $\begin{array}{c}\text { Factor } \\
\text { Autocon. }\end{array}$ & $\begin{array}{c}\text { factor II } \\
\text { Autorreal. }\end{array}$ & $\begin{array}{c}\text { Factor III } \\
\text { Autorres. }\end{array}$ & $\begin{array}{c}\text { Factor3 } \\
\text { Autoconf. }\end{array}$ & $\begin{array}{c}\text { Nivel } \\
\text { Diagn }\end{array}$ & Categoría \\
\hline $0-20$ & $0-29$ & $0-11$ & $0-13$ & $0-2$ & 0 & I & Aut.muy alta \\
$21-40$ & $30-33$ & $12-13$ & 14 & 3 & 1 & II & Aut.Baja \\
$41-60$ & $34-36$ & $14-15$ & $15-16$ & & 2 & III & Aut.Intermedia \\
$61-80$ & $37-38$ & & & 4 & & IV & Aut.Alta \\
$81-99$ & $39-41$ & 16 & 17 & 5 & 3 & V & Aut.muy alta \\
\hline
\end{tabular}

Tabla $N^{\circ} 9$

Baremo de ALPEL por edad 21-30 años

\begin{tabular}{|l|c|c|c|c|c|c|c|}
\hline Pc & $\begin{array}{l}\text { Autoestima } \\
\text { Total }\end{array}$ & $\begin{array}{l}\text { Factor } \\
\text { Autocon. }\end{array}$ & $\begin{array}{l}\text { factor II } \\
\text { Autorreal. }\end{array}$ & $\begin{array}{l}\text { Factor III } \\
\text { Autorres. }\end{array}$ & $\begin{array}{l}\text { Factor3 } \\
\text { Autoconf. }\end{array}$ & $\begin{array}{l}\text { Nivel } \\
\text { Diagn }\end{array}$ & Categoría \\
\hline $0-20$ & $0-29$ & $0-11$ & $0-13$ & $0-2$ & 0 & I & Aut.muy alta \\
$21-40$ & $30-33$ & $12-13$ & 14 & 3 & 1 & II & Aut.Baja \\
$41-60$ & $34-36$ & $14-15$ & $15-16$ & & 2 & III & Aut.Intermedia \\
$61-80$ & $37-38$ & & & 4 & & IV & Aut.Alta \\
$81-99$ & $39-41$ & 16 & 17 & 5 & 3 & V & Aut.muy alta \\
\hline
\end{tabular}

Tabla $\mathrm{N}^{\circ} 10$

Baremo de ALPEL por edad 41-mas años

\begin{tabular}{|l|c|c|c|c|c|c|c|}
\hline Pc & $\begin{array}{l}\text { Autoestima } \\
\text { Total }\end{array}$ & $\begin{array}{l}\text { Factor } \\
\text { Autocon. }\end{array}$ & $\begin{array}{l}\text { factor II } \\
\text { Autorreal. }\end{array}$ & $\begin{array}{l}\text { Factor III } \\
\text { Autorres. }\end{array}$ & $\begin{array}{l}\text { Factor3 } \\
\text { Autoconf. }\end{array}$ & $\begin{array}{l}\text { Nivel } \\
\text { Diagn }\end{array}$ & Categoría \\
\hline $0-20$ & $0-22$ & $0-1$ & $0-13$ & $0-2$ & 0 & I & Aut.muy alta \\
$21-40$ & $23-26$ & $2-5$ & 14 & 3 & 1 & II & Aut.Baja \\
$41-60$ & $27-35$ & $6-13$ & $15-16$ & & 2 & III & Aut.Intermedia \\
$61-80$ & $36-38$ & $14-15$ & & 4 & & IV & Aut.Alta \\
$81-99$ & $39-41$ & 16 & 17 & 5 & 3 & V & Aut.muy alta \\
\hline
\end{tabular}

Tabla $\mathbf{N}^{\circ} 11$

Baremo de ALPEL por estado civil-soltero

\begin{tabular}{|c|c|c|c|c|c|c|c|}
\hline Pc & $\begin{array}{c}\text { Autoestima } \\
\text { Total }\end{array}$ & $\begin{array}{c}\text { Factor } \\
\text { Autocon. }\end{array}$ & $\begin{array}{c}\text { factor II } \\
\text { Autorreal. }\end{array}$ & $\begin{array}{c}\text { Factor III } \\
\text { Autorres. }\end{array}$ & $\begin{array}{c}\text { Factor3 } \\
\text { Autoconf. }\end{array}$ & $\begin{array}{c}\text { Nivel } \\
\text { Diagn }\end{array}$ & Categoría \\
\hline $0-20$ & $0-29$ & $0-13$ & $0-13$ & $0-1$ & 0 & I & Aut.muy alta \\
$21-40$ & $12-13$ & 14 & 14 & 2 & 1 & II & Aut.Baja \\
$41-60$ & $14-15$ & $15-16$ & $15-16$ & 3 & 2 & III & Aut.Intermedia \\
$61-80$ & & & & 4 & & IV & Aut.Alta \\
$81-99$ & 16 & 17 & 17 & 5 & 3 & V & Aut.muy alta \\
\hline
\end{tabular}

$$
X=33.21 \quad \text { D.S. }=5.26 \quad N=2936
$$


Tabla $\mathbf{N}^{\circ} 12$

Baremo de ALPEL por estado civil-soltero

\begin{tabular}{|l|c|l|c|c|c|c|l|}
\hline Pc & $\begin{array}{l}\text { Autoestima } \\
\text { Total }\end{array}$ & $\begin{array}{l}\text { Factor } \\
\text { Autocon. }\end{array}$ & $\begin{array}{l}\text { factor II } \\
\text { Autorreal. }\end{array}$ & $\begin{array}{l}\text { Factor III } \\
\text { Autorres. }\end{array}$ & $\begin{array}{l}\text { Factor3 } \\
\text { Autoconf. }\end{array}$ & $\begin{array}{l}\text { Nivel } \\
\text { Diagn }\end{array}$ & Categoría \\
\hline $0-20$ & $0-23$ & $0-2$ & $0-2$ & $0-2$ & 0 & I & Aut.muy alta \\
$21-40$ & $24-31$ & $3-11$ & 3 & 3 & 1 & II & Aut.Baja \\
$41-60$ & $32-36$ & $12-15$ & & & 2 & III & Aut.Intermedia \\
$61-80$ & $37-39$ & & 4 & 4 & & IV & Aut.Alta \\
$81-99$ & $40-41$ & 16 & 5 & 5 & 3 & V & Aut.muy alta \\
\hline
\end{tabular}

\section{BIBLIOGRAFIA}

Aiken, L. R. Test psicológicos y evaluación. México, Edit. Prentice Hall International, 1994.

Brown, F. Principios de la medición en psicología y educación. México, Edit. Manual Moderno, 1996.

Curvin, R. Como fomentar valores individuales. Barcelona, España, Ed. CEAC, 1989.

Cruz Ramirez, J. Autoestima y gestión de calidad. México, Grupo editorial Iberoamericana,1996.

Garret, H. E. Estadística en psicología y educación. Argentina, Ed. Paidós, 1974.

Kline, P. The Handbook of psichological testing. London, Routhledge, 1993.

Loli, A. Y Lopez, E. La autoestima y los valores en la calidad y la excelencia. Lima, UNMSM,I997.

Loli, A. Y Lopez, E. La autoestima y los valores en las microempresas del Cono este de Lima. Perú, UNMSM,1998.

Loli, A. Y Lopez, E. La autoestima y los valores organizacionales en estudiantes universitarios. Lima, Perú, UNMSM,1999.

Marin, G. Manual de investigación en psicología social. México, Ed.Trillas, 1975.

Maslow, A. El hombre autorrealizado. Ed. Kairós, Barcelona, 1979.

Muñiz, J. Teoría clásica de los test. Madrid, Edit. Pirámide, 1994.

Muñiz, J. Psicometría. Madrid, Edil. Universitas S.A., 1996.

Peters T. y Waterman, R. En busca de la excelencia. Colombia, Ed. NORMA, 1996.

Rodriguez, M. Como desarrollar la autoestima. México, Instituto de Desarrollo Humano, s/f.

Rodriguez, M. Los valores clave de la excelencia. México, McGraw Hill, 1993.

Siegel, S. y Castelan, N. Estadística no paramétrica: Aplicada a las ciencias de la conducta. México, Edit.Trillas, 1995. 